\title{
Influência do déficit de estatura nos desvios nutricionais em adolescentes e pré-adolescentes
}

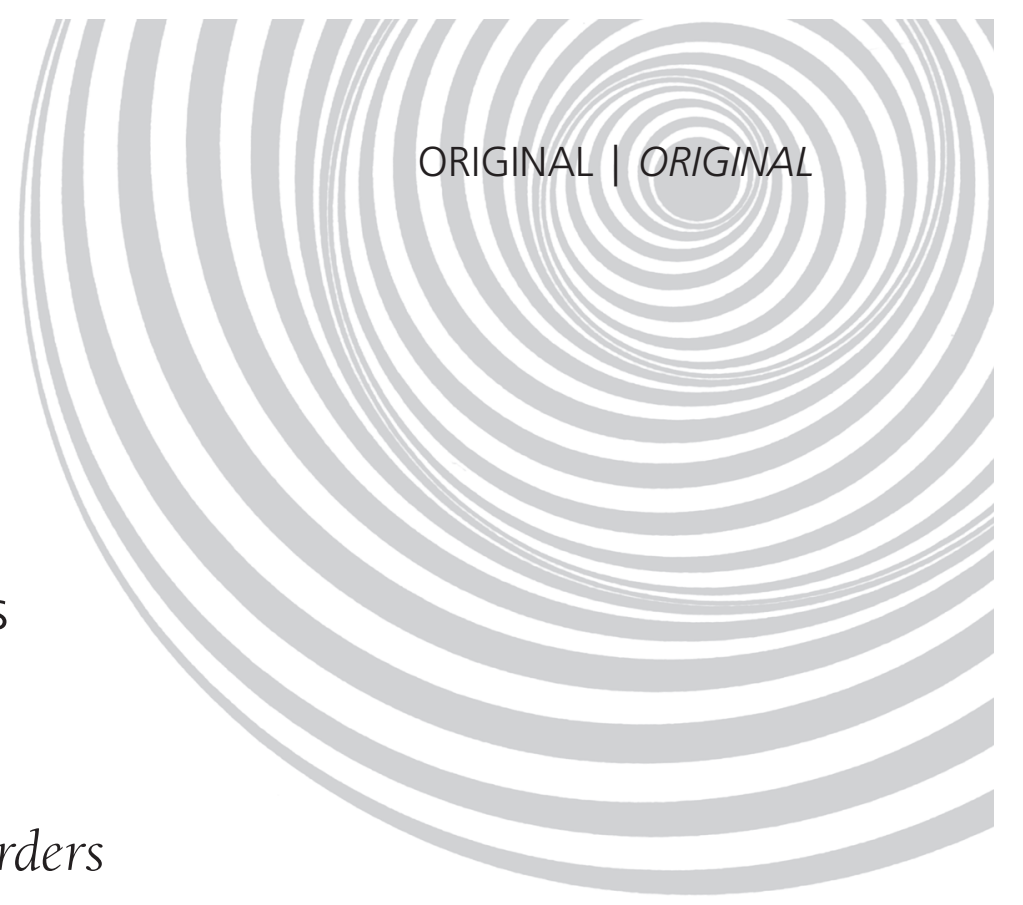

\section{Influence of stunting on nutrition disorders in adolescents and pre-adolescents}

Carla Danusa da Luz SANTOS'

Ana Paula Grotti CLEMENTE ${ }^{2}$

Paula Andrea MARTINS 3

Ana Lydia SAWAYA ${ }^{4}$

RE S U M O

\section{Objetivo}

Avaliar a influência do déficit estatural nos desvios nutricionais em pré-adolescentes e adolescentes de baixa renda.

\section{Métodos}

Calculou-se o Índice de Massa Corporal a partir do peso e da estatura de alunos da rede pública entre 4 e $8^{a}$ séries. Foram utilizadas as tabelas do National Center for Health Statistics de 2000 para crianças e adolescentes e foi considerado excesso de peso Índice de Massa Corporal/ldade $\geq$ P85 e déficit de peso Índice de Massa Corporal/ldade $\leq$ P5. Déficit estatural foi classificado quando estatura/idade foi menor que -1 desvio-padrão. Foram comparadas as proporções de baixo peso e excesso de peso nos grupos com e sem déficit estatural, de acordo com sexo e faixa etária.

\section{Resultados}

Foram avaliados 3766 jovens sendo 50,4\% do sexo feminino. A média de idade foi de 12,8, desvio-padrão de 2,0 anos, sem diferença significante entre os grupos. No grupo com déficit de estatura, observou-se que o

\footnotetext{
1 Universidade Federal de São Paulo, Pós-Graduação em Pediatria, Departamento de Pediatria, Disciplina de Fisiologia da Nutrição. R. Botucatu, 598, Vila Clementino, 04023-062, São Paulo, SP, Brasil. Correspondência para/Correspondence to: C.D.L. SANTOS.E-mail: <carladanusa@ecb.epm.br>.

2 Universidade Federal de São Paulo, Pós-Graduação em Endocrinologia, Disciplina de Fisiologia da Nutrição. São Paulo, SP, Brasil.

3 Universidade Federal de São Paulo, Curso de Nutrição, Departamento de Ciências da Saúde. Campus Baixada Santista. Santos, SP, Brasil.

${ }^{4}$ Universidade Federal de São Paulo, Departamento de Fisiologia. São Paulo, SP, Brasil.

Apoio: Fundação de Amparo à Pesquisa do Estado de São Paulo, Coordenação de Aperfeiçoamento de Pessoal de Nível Superior e International Atomic Energy Agency.
} 
188 | C.D.L. SANTOS et al.

excesso de peso é mais prevalente entre os mais velhos. Naqueles sem déficit de estatura a distribuição por idades foi semelhante para as três classificações de Índice de Massa Corporal/Idade. A distribuição por sexo mostra que o excesso de peso no grupo com déficit de estatura foi mais acentuada entre as meninas $(76,1 \%)$ enquanto que no grupo com estatura normal a diferença foi mínima entre os sexos $(4,4 \%)$.

\section{Conclusão}

Na presença do déficit de estatura, o excesso de peso foi mais prevalente nos maiores de 12 anos e entre o sexo feminino. Os meninos sem déficit de estatura mostraram uma proporção maior de excesso de peso em relação aos com déficit de estatura.

Termos de indexação: Adolescente. Deficiências nutricionais. Estatura.

\section{A B S T R A C T}

\section{Objective}

The objective of this study was to evaluate the influence of stunting on nutrition disorders in low-income preadolescents and adolescents.

\section{Methods}

Body mass index was calculated from the weight and height of 4 to $8^{\text {th }}$ grade students from public schools. The tables from the National Center for Health Statistics of 2000 for children and adolescents were used as reference guides. Individuals were considered overweight when Body Mass Index-for-age $\geq P 85$ and underweight when Body Mass Index-for-age $\leq P 5$. Individuals were considered stunted when height-for-age was below one standard deviation. Overweight and underweight rates were compared between the non-stunted and stunted groups according to gender and age.

\section{Results}

A total of 3766 individuals were studied, $50.4 \%$ being females. Mean age was 12.8 years, with a standard deviation of 2.0 years and no significant difference between the groups. In the stunted group, overweight was more common among the older children. In the non-stunted group, the distribution by age was similar for all three Body Mass Index-for-age classifications. Distribution by gender shows that overweight in the stunted group prevailed among females (76.1\%) while in the non-stunted group the difference was minimal between the genders (4.4\%).

\section{Conclusion}

In the stunted group, overweight was more common among those older than 12 years and among females. Non-stunted boys were overweight more often than stunted boys.

Indexing terms: Adolescent. Body height. Deficiency diseases.

\section{N T R O D U Ç Ã O}

O indicador estatura/idade (E/I) mede o crescimento linear e seu déficit relaciona-se a alterações cumulativas de longo prazo na situação nutricional e de saúde em geral. Estando associado a processos de longa duração e a um acumulado retardo de crescimento, reflete formas crônicas de desnutrição ${ }^{1}$. O crescimento e a estatura final de um indivíduo dependem, fundamentalmente, da interação entre seu potencial genético e a influência de um grande número de fatores ambientais. No entanto, nessa interação, os fatores ambientais assumem importância primordial, especialmente nos países em desenvolvimento, onde as diferenças genéticas parecem ser menos importantes do que diferenças no meio ambiente ${ }^{1-3}$. Entre os fatores ambientais, condições precárias de habitação e saneamento, baixos níveis de renda e baixa escolaridade dos pais têm sido apontados como fatores determinantes do déficit estatural ${ }^{4-6}$. Estima-se que 38,1\% das crianças menores de cinco anos que vivem em países em desenvolvimento padeçam de comprometimento grave do crescimento ${ }^{7}$. No Brasil, a estimativa mais recente da prevalência de baixa 
estatura em adolescentes é de 10\% sendo maior a freqüência nos meninos $(11,3 \%)$ do que nas meninas $(8,3 \%)^{8}$. Em ambos os sexos, a freqüência de déficit de estatura diminui com o aumento da renda familiar: cerca de 20,0\% quando a renda é de até 0,5 salário-mínimo per capita e 4-5\% quando a renda é de 5 ou mais salários-mínimos per capita ${ }^{8}$.

Diversos estudos no mundo têm mostrado que a desnutrição no início da vida pode, no futuro, associar-se à obesidade e às enfermidades a ela associadas. Elevado quociente respiratório (QR) (maior oxidação dos carboidratos), baixa taxa de oxidação lipídica, aumentada relação cintura/quadril (RCQ) foram encontrados em meninos e meninas com baixa estatura,10. Maior acúmulo de gordura subcutânea e maior prevalência de excesso de peso também já foram associados à baixa estatura ${ }^{11,12}$.

A baixa estatura também já se mostrou associada à hipertensão em adultos, especialmente entre mulheres ${ }^{10-13}$ e em adolescentes ${ }^{14}$. A regulação da glicose e insulina também parece sofrer alteração na vigência de déficit nutricional em fases críticas do desenvolvimento. Função das células beta pancreáticas diminuída e aumento da sensibilidade à insulina foram encontrados em crianças com baixa estatura nutricional ${ }^{15}$ e curvas mais elevadas de glicose e insulina foram observadas em homens jovens com história de desnutrição no primeiro ano de vida ${ }^{16}$.

Estas alterações provavelmente estão envolvidas nos mecanismos pelos quais a desnutrição energético-protéica nos primeiros anos de vida provoca modificações metabólicas responsáveis pelo aparecimento de doenças crônicas na vida adulta. No entanto, parece que durante a adolescência deve ocorrer a precipitação de tais manifestações que se tornam mais evidentes na fase adulta. No Brasil, poucos são os estudos que avaliam o impacto do déficit de estatura no ganho de peso nessa faixa etária. A maioria dos estudos traça um perfil do estado antropométrico sem, no entanto, levar em consideração o crescimento linear. Uma vez que diversas alterações meta- bólicas já foram evidenciadas na presença de déficit de estatura, fica evidente a necessidade de identificar precocemente os desvios nutricionais que incidem nessa população. Diante disso, o objetivo deste trabalho foi avaliar a influência do déficit estatural nos desvios nutricionais em pré-adolescentes e adolescentes estudantes de escolas da rede pública.

\section{MÉTODOS}

Trata-se de um estudo transversal, composto por adolescentes de 9 escolas da rede pública (entre $4^{\mathrm{a}}$ e $8^{\mathrm{a}}$ séries) e freqüentadores de 4 Núcleos Sócio Educativos da zona sul do município de São Paulo (população de baixa renda).

A escolha das instituições participantes foi feita de forma aleatória entre aquelas localizadas nas proximidades ou dentro de favelas da zona sul do município de São Paulo. A coleta dos dados passou por uma etapa prévia de esclarecimento para a direção e os professores, sobre os procedimentos que seriam realizados e os objetivos do estudo. Os pais dos alunos foram informados sobre a pesquisa por uma carta circular e autorizaram a participação dos filhos por assinatura do termo de consentimento livre e informado.

O peso foi obtido em única tomada com balança tipo plataforma com capacidade de $150 \mathrm{~kg}$ e precisão de $100 \mathrm{~g}$. A aferição da estatura foi obtida por um único examinador treinado utilizando antropômetro portátil modelo AlturExata, conforme as normas padronizadas ${ }^{17}$. Os dados foram digitados no programa Epi Info versão 6.2 para cálculo dos indicadores antropométricos. O Índice de Massa Corporal (IMC) foi calculado dividindo-se o peso em quilogramas pelo quadrado da estatura em metros. Como padrão de referência foram usadas as tabelas do National Center for Health Statistics (NCHS) (2000) para crianças e adolescentes, de acordo com sexo e idade $^{18}$. A classificação nutricional considerou os critérios da Organização Mundial da Saúde $(\mathrm{OMS})^{4}$ : déficit estatural grave quando E/I foi menor que-3DP, moderada entre-2 e-3DP e leve 
entre -1 e -2DP. Foi considerado excesso de peso IMC/I $\geq P 85$ e déficit de peso IMC/ISP5.

Para as análises foi utilizado o software SPSS versão 13. A população foi agrupada em: déficit de estatura (quando E/I foi menor ou igual a -1DP) e estatura normal (quando E/l foi maior que -1DP). Para cada um dos grupos foi feita comparação da distribuição das faixas etárias por categorias de IMC utilizando o teste do qui-quadrado. Utilizando o mesmo teste, também foi feita comparação por sexo segundo categorias de IMC para cada um dos grupos (déficit de estatura e estatura normal). Considerando que a idade da população apresentou distribuição normal, foi usado Teste $t$ para comparação de médias de idade entre os grupos. Para rejeição da hipótese nula, considerou-se o nível de significância de 5\%.

Este estudo foi aprovado pelo Comitê de Ética em pesquisa da Universidade Federal de São Paulo (Protocolo no 0617/06), observando-se o cumprimento dos princípios éticos contidos na Declaração de Helsinki, da Word Medical Association.
RES U LTA D OS

Todas as instituições convidadas aceitaram participar do estudo. A perda amostral por não comparecimento ou recusa foi de 380 adolescentes. Por fim, foram avaliados 3766 jovens, sendo $50,4 \%$ foi do sexo feminino e $49,6 \%$ do sexo masculino. A média de idade foi de 12,8 , $\mathrm{DP}=2,0$ anos, sem diferença estatisticamente significante $(p=0,189)$ entre os grupos déficit de estatura (E/IS-1) e estatura normal (E/l>-1). Segundo a distribuição por faixa etária, 6,0\% tinham entre 8 e 9 anos, 33,0\% entre 10 e 11 anos, $46,8 \%$ entre 12 e 14 anos, $13,1 \%$ entre 15 e 17 anos e 1,1\% entre 18 e 19 anos. A avaliação do índice $E / I$ mostrou freqüência de déficit de estatura em 20,1\% dos jovens, sendo baixa estatura moderada e grave de $3,7 \%,(n=139)$ e déficit leve de estatura $16,4 \%(n=617)$. A proporção de baixo e excesso de peso entre aqueles com déficit de estatura (E/IS-1) foi de 10,3 e 9,4\%, respectivamente enquanto que para aqueles com estatura normal, essas proporções foram de 4,2 e 19,8\% (Tabela 1).

Tabela 1. Distribuição de escolares da rede pública, segundo classificação de estatura/idade e Índice de Massa Corporal/ldade ( $n=3$ 766). São Paulo (SP), 2007.

\begin{tabular}{|c|c|c|c|c|c|c|}
\hline \multirow{2}{*}{ Estado nutricional (IMC/I) } & \multicolumn{2}{|c|}{ Déficit de estatura } & \multicolumn{2}{|c|}{ Estatura normal } & \multicolumn{2}{|c|}{ Total } \\
\hline & $n$ & $\%$ & $n$ & $\%$ & $n$ & $\%$ \\
\hline Baixo peso & 78 & 10,3 & 125 & 4,2 & 203 & 5,4 \\
\hline Eutrófico & 607 & 80,3 & 2287 & 76,0 & 2894 & 76,8 \\
\hline Excesso de peso & 71 & 9,4 & 598 & 19,8 & 669 & 17,8 \\
\hline Total & 756 & 100,0 & 3010 & 100,0 & 3766 & 100,0 \\
\hline
\end{tabular}

Estatura normal: E/I >-1DP; déficit de estatura: E/I <-1; baixo peso: IMC/Ispercentil 5; eutrófico: IMC/l>percentil 5 e IMC/l<percentil 85; excesso de peso: IMC/I $\geq$ percentil 85 .

Tabela 2. Estado nutricional de escolares da rede pública, segundo indicadores estatura/idade, Índice de Massa Corporal/Idade e sexo. (n=3 766). São Paulo (SP), 2007.

\begin{tabular}{|c|c|c|c|c|c|c|}
\hline \multirow{2}{*}{ Estado nutricional (IMC/I) } & \multicolumn{3}{|c|}{ Déficit de estatura*(\%) } & \multicolumn{3}{|c|}{ Estrutura normal**(\%) } \\
\hline & $\mathrm{F}$ & $M$ & Total & $\mathrm{F}$ & $M$ & Total \\
\hline Baixo peso & 37,2 & 62,8 & 100,0 & 40,8 & 59,2 & 100,0 \\
\hline Eutrófico & 50,4 & 49,6 & 100,0 & 51,2 & 48,8 & 100,0 \\
\hline Excesso de peso & 76,1 & 23,9 & 100,0 & 47,8 & 52,2 & 100,0 \\
\hline
\end{tabular}

* $\chi^{2}$ (teste qui-quadrado)=23,831; $2 \mathrm{gl}$ (graus de liberdade); $p<0,001 ;$ nível de significância $\alpha=0,05 ;{ }^{* *} \chi^{2}$ (teste qui-quadrado)=6,673; $2 \mathrm{gl}$ (graus de liberdade); $p=0,036$; nível de significância $\alpha=0,05$. 


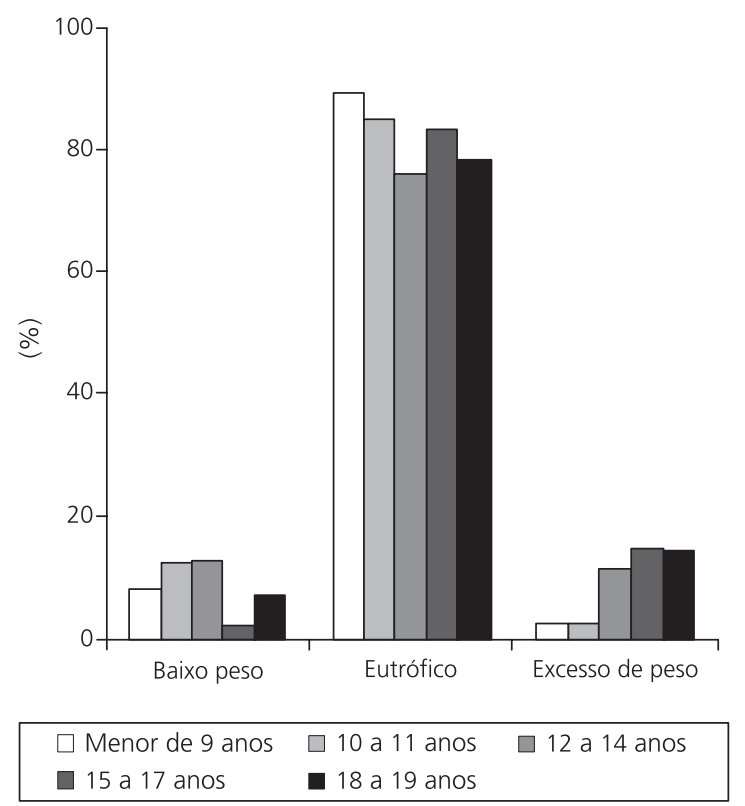

Figura 1. Caracterização de escolares da rede pública, com déficit de estatura segundo indicador Índice de Massa Corporal/Idade e faixa etária. São Paulo (SP), 2007.

Nota: $\chi^{2}$ (teste qui-quadrado)=31,516; $8 \mathrm{gl}$ (graus de liberdade); $p<0,001$; nível de significância $\alpha=0,05$.

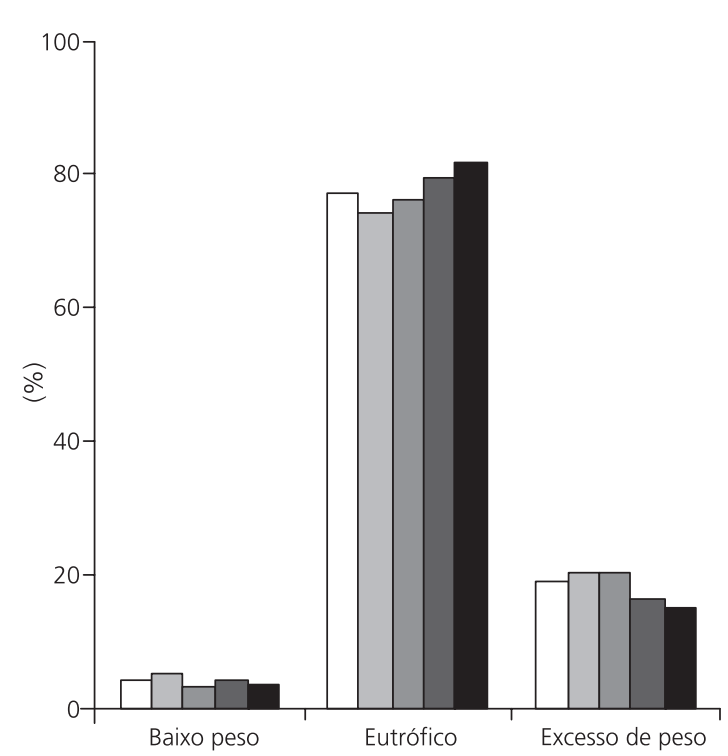

\begin{tabular}{|ll|}
\hline $\begin{array}{l}\text { Menor de } 9 \text { anos } \\
\square 10 \text { a } 11 \text { anos }\end{array}$ & $\square 12$ a 14 anos \\
$\square$ a 17 anos & $\square 18$ a 19 anos
\end{tabular}

Figura 2. Caracterização de escolares da rede pública, com estatura normal segundo indicador Índice de Massa Corporal/ldade e faixa etária. São Paulo (SP), 2007.

Notas: $\chi^{2}$ (teste qui-quadrado) $=8,427 ; 8 \mathrm{gl}$ (graus de liberdade); $p<0,393$; nível de significância $\alpha=0,05$.
Segundo a classificação de E/l por faixa etária, no grupo com déficit de estatura (E/IS-1), a distribuição por idade é diferente para cada uma das categorias de IMC $(p<0,001)$. Observa-se neste grupo maior proporção do excesso de peso (IMC/I $\geq 85$ ) nos maiores de 12 anos (Figura1). Naqueles sem déficit de estatura a distribuição por idades foi semelhante para as três classificações de IMC/I $(p=0,393)$ (Figura2). Na distribuição por sexo, houve diferença estatisticamente significante tanto no grupo com déficit de estatura $(p<0,001)$ quanto no grupo com estatura normal $(p=0,036)$. Nos 2 grupos, a proporção de baixo peso foi maior no sexo masculino. Quanto ao excesso de peso, no grupo com déficit de estatura a proporção foi consideravelmente maior entre as meninas $(76,1 \%)$ enquanto que no grupo com estatura normal a diferença de proporção entre os sexos foi de apenas 4,4\% (Tabela 2).

\section{DISCUSSÃO}

Dados brasileiros com relação à avaliação nutricional na adolescência são ainda limitados e a maioria dos estudos não leva em consideração o crescimento linear. Além disso, a diversidade de critérios utilizados na classificação de sobrepeso e obesidade dificulta a comparação entre estudos antropométricos. Nesse sentido, o presente estudo contribuiu para mostrar que a presença de déficit de estatura determina diferenças na distribuição do IMC/I segundo faixas de idade e também entre os sexos, levando à maior proporção de sobrepeso em meninas.

O presente estudo considerou o nível de corte de -1 escore-Z para o indicador E/l que classifica desnutrição leve ${ }^{7}$ para investigar se os adolescentes com leve déficit de estatura já apresentariam uma tendência maior ao excesso de peso em relação àqueles com estatura normal. Para classificar o grupo com déficit de estatura, não foi levada em consideração a estatura dos pais. Muitas publicações têm mostrado que o padrão genético somente tem sido útil como bom preditor da estatura das crianças quando se trata 
de nível socioeconômico alto, quando o potencial de crescimento pode ser totalmente expresso ${ }^{19,20}$. Nos níveis socioeconômicos baixos, a baixa estatura dos pais não permite excluir a possibilidade de que a baixa estatura destes resulte do efeito acumulado da pobreza em várias gerações da família.

Em ambos os sexos, no grupo de jovens com déficit de estatura as proporções de baixo e excesso de peso foram semelhantes, sendo que o baixo peso foi duas vezes maior, comparado àqueles com estatura normal. Isso mostra que a inadequação alimentar, que provavelmente conduziu ao déficit de crescimento, continua presente. Não foi possível coletar as variáveis necessárias para definir adequadamente a condição socioeconômica da população. Contudo, a localização das instituições sendo próximas a favelas, possivelmente permitiu a inclusão de jovens moradores de domicílios com elevado grau de carências socioeconômicas.

Outro dado que chama atenção é o comportamento do excesso de peso naqueles com déficit de estatura. Apesar de este ser quase a metade do total de excesso de peso apresentado por aqueles com estatura normal, o grupo com déficit de estatura apresenta maiores proporções de excesso de peso entre os adolescentes mais velhos. Um estudo com crianças africanas que tiveram diagnóstico de baixa estatura aos 2 anos (E/l<-2 escores-Z) não encontrou relação significante entre baixa estatura e composição corporal aos 9 anos $^{21}$. Os autores ressaltam que esses achados não descartam a possibilidade de essas crianças com baixa estatura apresentarem sobrepeso ou obesidade durante a adolescência, mas que o estudo foi incapaz de mostrar essas alterações antes do início do desenvolvimento puberal. Os dados do presente estudo sugerem que na presença do déficit de estatura, os jovens mais velhos apresentaram maiores proporções de sobrepeso. Por outro lado, um estudo com crianças e adolescentes brasileiros de 7 a 19 anos, sem considerar o crescimento linear, observou que as prevalências de sobrepeso e obesidade diminuíram à medida que ocorreu aumento da faixa etária ${ }^{22}$. Esse seria um fenômeno esperado, pois se sabe que um moderado excesso de peso poderá ser compensado pelo futuro crescimento. Os levantamentos nacionais também avaliam o IMC/I sem considerar o crescimento linear e, nesse caso, o último levantamento mostrou redução do IMC/I com o avançar da idade. Em ambos os sexos, a freqüência do excesso de peso foi máxima entre adolescentes de 10-11 anos (cerca de 22\%), diminuindo para 12-15\% no final da adolescência ${ }^{8}$, diferentemente dos dados deste trabalho, que mostraram que na ausência de déficit de crescimento, não houve alteração do percentil de IMC/I com o avançar da idade.

O IMC, apesar de ser um bom indicador para avaliar adiposidade, não quantifica a composição corporal. Embora o presente estudo não tenha o objetivo de avaliar a composição corporal dos jovens, é possível que o elevado IMC/I observado entre aqueles com déficit estatural também venha acompanhado pelo incremento de tecido adiposo, principalmente entre o sexo feminino como tem sido apontado na literatura ${ }^{23}$. Uma coorte com adolescentes senegalesas com história de baixa estatura na infância não mostrou diferenças de IMC entre os grupos com e sem história de baixa estatura, porém mostrou que aquelas que tinham história de baixa estatura apresentaram maiores dobras cutâneas biciptal e triciptal do que o outro grupo sem história de baixa estatura ${ }^{11}$. Recentemente, um estudo que acompanhou durante 3 anos meninas e meninos moradores de favelas de 11-15 anos, mostrou que os meninos com déficit de estatura (E/l<-1,5 escore-Z) acumularam mais massa gorda (\%) e ganharam menos massa magra ( $\mathrm{kg} \mathrm{e} \mathrm{\% ),} \mathrm{enquanto} \mathrm{que} \mathrm{aqueles}$ com estatura normal apresentaram diminuição da gordura corporal com o tempo. No mesmo estudo, as meninas com déficit de estatura também ganharam menos massa magra e apresentaram tendência em aumentar o percentual de gordura corporal $^{15}$. 


\section{O N CLUSÃ O}

Os dados do presente estudo mostram que a proporção de baixo peso nos adolescentes estudados é duas vezes maior na presença de déficit de estatura, mostrando continuação do agravo nutricional que, provavelmente, determinou déficit no crescimento linear durante a infância. A proporção do excesso de peso naqueles com déficit de estatura, foi maior entre os mais velhos e no sexo feminino, fato não observado no grupo com estatura normal. Novos estudos tornam-se necessários para avaliar em maior profundidade a influência do início da puberdade no ganho de peso, gordura corporal e demais fatores associados ao desenvolvimento da síndrome metabólica em adolescentes com déficit de estatura de causa nutricional.

\section{OLABORAÇÃ O}

C.D.L. SANTOS e A.L. SAWAYA participaram na elaboração do projeto de pesquisa, na coleta de dados na tabulação e discussão dos resultados e na redação. A.P.G. CLEMENTE participou na elaboração do projeto de pesquisa, na coleta de dados, na tabulação e discussão dos resultados. P.A. MARTINS participou na tabulação dos dados.

\section{REFERÊ N CIAS}

1. Waterlow JC. Protein-energy malnutrition the nature and extent of the problem. Clin Nutr. 1997; 16(Suppl 1):3-9.

2. Amigo $H$, Bustos P. Factores de riesgo de talla baja en escolares chilenos de zonas rurales de alta vulnerabilidade social. Arch Latinoam Nutr. 1995; 45(2):97-102.

3. Kac G. Tendência secular em estatura: uma revisão da literatura. Cad Saúde Pública. 1999; 15(3): 451-61.

4. World Health Organization. Measuring change in nutritional status. Geneva; 1983.

5. Lei DLM, Freitas IC, Chaves SP, Lerner BR, Stefanini $\mathrm{ML}$. Linear growth retardation and social factors among schoolchildren from the city of Osasco, São Paulo, Brazil. Cad Saúde Pública. 1997; 13(2): 277-83.
6. Guimaraes LV, Latorre MRD, Barros MBA. Risk factors for short stature in preschool children. Cad Saúde Pública. 1999; 15(3):605-15.

7. World Health Organization. Report of a WHO. Global database on child growth and malnutrition. Geneva; 1997.

8. Instituto Brasileiro de Geografia e Estatística. Pesquisa de orçamentos familiares 2002-2003. Análise da disponibilidade domiciliar de alimentos e do estado nutricional no Brasil. Rio de Janeiro; 2004.

9. Hoffman DJ, Sawaya AL, Verreschi I, Tucker KL, Roberts S. Why are nutritionally stunted children at increased risk of obesity? Studies of metabolic rate and fat oxidation in shantytown children from São Paulo, Brazil. Am J Clin Nutr. 2000; 72(3): 702-7.

10. Ferreira HS, Florêncio TMTM, Fragoso MAC, Melo FP, Silva TG. Hipertensão, obesidade abdominal e baixa estatura: aspectos da transição nutricional em uma população favelada. Rev Nutr. 2005; 18(2): 209-18.

11. Benefice E, Garnier D, Simondon KB, Malina RM. Relationship between stunting in infancy and growth and fat distribution during adolescence in Senegalese girls. Eur J Clin Nutr. 2001; 55(1):50-8.

12. Florêncio TM, Ferreira HS, Cavalcante JC, Luciano SM, Sawaya AL. Food consumed does not account for the higher prevalence of obesity among stunted adults in a very low-income population in the Northeast of Brazil (Maceió, Alagoas). Eur J Clin Nutr. 2003; 57(11):1437-46.

13. Sichieri R, Siqueira KS, Pereira RA, Ascherio A. Short stature and hypertension in the city of Rio de Janeiro, Brazil. Public Health Nutr. 2000; 3(1): 77-82.

14. Fernandes MTB, Sesso S, Martins PA, Sawaya AL. Increased blood pressure in adolescents of low socioeconomic status with short stature. Pediatr Nephrol. 2003; 18(5):435-9.

15. Martins PA, Sawaya AL. Evidence for impaired insulin production and higher sensitivity in stunted children living in slums. Br J Nutr. 2006; 95(5): 996-1001.

16. Gonzalez JB, Rios JMT, Castillo LM, López JCA, Aquilar CAS, Bouchard $C$, et al. Effect of malnutrition during the first year of life on adult plasma insulin and glucose tolerance. Metabolism. 2003; 52(8):1005-11.

17. Lohman T. Advances in body composition assessment: current issues. Medicine science [monograph 3]. Champaign IL: Kinetics Publishers; 1992. 
194 | C.D.L. SANTOS et al.

18. Centers for Disease Control and Prevention, National Center For Health Statistics. 2000 CDC growth charts: United States [online]. Hyaltsville; 2002 [cited 2002 May 11]. Available from: <http:// www.cdc.gov/growthcharts>.

19. Amigo H, Bustos P. Risk factors of short stature in Chilean school children from rural areas of high social vulnerability. Arch Latinoam Nutr. 1995; 45(2):97-102.

20. Voss LD, Mulligan J, Betts PR. Short stature at school entry: an index of social deprivation? Child Care Health Dev. 1998; 24(2):145-56.

21 Cameron N, Wright MM, Griffiths PL, Shane A. Stunting at 2 years in relation to body composition

at 9 years in African urban children. Obes Res. 2005; 13(1):131-6.

22. Silva GAP, Balaban G, Motta MEFA. Prevalência de sobrepeso e obesidade em crianças e adolescentes de diferentes condições socioeconômicas. Rev Bras Saúde Mater Infant. 2005; 5(1):53-9.

23. Sawaya AL, Grillo LP, Verreschi I, Silva AC, Roberts SB. Mild stunting is associated with higher susceptibility to the effects of high fat diets: studies in a shantytown population in São Paulo, Brazil. J Nutr. 1998; 128(Suppl 2):415-20.

Recebido em: 6/8/2007

Versão final reapresentada em: 11/3/2008 Aprovado em: 11/7/2008 